\title{
MultiDisciplinary Design for Uninhabited Air Vehicles
}

\author{
Max Blair, Steven R. LeClair, Jeffrey V. Zweber, Adel Chemaly \\ Wright Laboratory \\ Wright-Patterson AFB, OH 45433-7542
}

\begin{abstract}
Contemporary product design and process development is based on an iterative specify-evaluate-revise approach which is often time intensive and therein non-responsive to customer needs. The engineering of a product incorporates numerous stages involving conceptual through detailed design. For small quantities or lot sizes (1-25 parts), the product design and process planning steps account for an inordinate share of the overall development cycle. An integrated geometric, performance analysis, and materials processing evaluation environment for concurrent, multidisciplinary design is needed to not only address design-through-production efficiencies and costs but enable more responsive product development cycles. The environment should enable the user to interactively evaluate alternative designs, not only of individual components or subsystems but combinatorial alternatives varying materials, performance envelopes and sizing. All of these issues are particularly relevant to a new class of aircraft, an uninhabited air vehicle (UAV), which is both unique in terms of its mission as well as vehicle life, environmental constraints, and component through system reliability and maintainability.
\end{abstract}

\section{TERMINOLOGY}

Dependency Tracking: All model variables know which other model variables influence them and which quantities they influence.

Demand-Driven Calculations: Quantities are only calculated when they are needed. This is in contrast to serial programming, where the analysis proceeds according to a preprogrammed set of instructions.

Full Geometric Associativity: The results of geometric design changes at the layout and component level are immediately reflected at the detailed part level.

Adaptive Modeling Language is a trademark of TechnoSoft Inc. Pro/ENGINEER is a registered trademark of Parametric Technology Corp. All other company and product names are trademarks or registered trademarks of their respective owners
Design Feed Forward: Design decisions and intention generated at the global level are reflected in subsequent levels of increasing detail.

Design Feed Back: Data which was generated at the detailed level is collected or parameterized to help guide decisions made at the global level.

Parametric Relations: Design variables can be reduced in number and controlled in meaningful parametric relations. One example is an empirical parametric relation between production cost and vehicle weight.

Conceptual Design: Work at the global level in order to discover and characterize the feasible design space for the overall vehicle configuration.

Preliminary Design: An increased number of specialized design variables used to address technical disciplinary issues (e.g. structures, aeroelasticity, controls etc.).

Detailed Design: Work within the requirements set forth by the preliminary designer to generate high fidelity design data.

\section{INTRODUCTION}

To address the issues of a needed integrated product-process design environment, reference will be made to the Adaptive Modeling Language ${ }^{\mathrm{TM}}$ (AML) architecture which has evolved from an in-house feature-based design project to a commercial product in use by industries ranging from automotive, e.g., Ford Motor and Volvo; to aerospace, e.g., Lockheed-Martin, and McDonnell-Douglas; and power generation, e.g., Zurn Balke-Durr and Siemens. AML, as described herein, supports a multidisciplinary environment for interactive product-process design by means of a sophisticated feature-based design environment, enabling the user to interactively propagate constraints across several modeling systems.

The FBDE (Feature Based Design Environment) within AML is representative of the capabilities of most parametric CAD systems, with tools for interactive feature dimensioning, positioning, and orientation. The interaction to be discussed here extends this conventional use of features to encompass combat aircraft synthesis; wherein models of 
vehicle sizing and component stiffness/loads bi-directionally constrain each other and the resultant vehicle design. Therein, features represent conceptual vehicle shapes with associated performance, sizing, and maneuvering constraints that enable the transfer of model results without transferring the geometry instance.

AML incorporates a unique underlying object-oriented model for representing geometric and non-geometric features to support bi-directional constraint propagation across multiple design disciplines. Such interaction is supported between geographically dispersed teams of scientists and engineers involving experts in flight dynamics, materials and manufacturing to interactively design a new vehicle.

The aerospace industry is painfully aware that the USAF is working to control the spiraling cost of new flight vehicles. There are a number of on-going strategies in which cost control might be realized. For example, Wright Laboratory (WL) is teaming with industry to direct both the Composite Affordability Initiative and the Lean Aircraft Initiative. Design process technology has been identified as a potential contributor in many of these strategies.

The Flight Dynamics Directorate (WL/FI) in the Wright Laboratory has populated its MultiDisciplinary Design Integrated Product Team (MDD/IPT) with a number of research aerospace engineers who understand air vehicle design. The key to our ultimate success in ongoing participation by the Materials Process Design Research Team (WL/MLIM) in Wright Laboratory. The MDD/IPT has a long range vision for addressing simultaneous cost and performance constraints with feed forward and feed back. Data will feed forward from conceptual design to control the detailed design. High fidelity data will feed back to inform the conceptual designer of the design consequences.

TechnoSoft Inc. markets the Adaptive Modeling Language which has taken a Knowledge-Based Approach to develop automated Design-for-Production processes. AML includes a proven suite of fully associative design objects which address issues of geometry, manufacturing, meshing and computational process.

The MDD/IPT, WL/MLIM and TechnoSoft are collaborating to demonstrate a design process with feed forward and feed back. In other words, we will simultaneously address both design-for-performance and design-for-production. In this paper, we will use AML to demonstrate a simple design thread between the global conceptual design and the preliminary design weight of a composite component.

Air vehicles have always been designed with cost and performance constraints. However, it is a common complaint that the long time lag between a performance motivated design change and the high fidelity feed back of stress, weight and cost consequences is impractical. The idea here is to model the complex relationships between detailed data (gathered from the manufacturing design) and parametric design data (at the conceptual level). Any relationship which can be automated, speeds up the redesign process and the gathering of design sensitivities.

This demonstration of concurrent engineering uses a conceptual model to control the configuration at the preliminary level. Going one step further, the preliminary model is used to provide high fidelity data to the conceptual designer. Thus, the designer is provided a design laboratory with near real-time feed back of the model and real-time feed back of design consequences. Since the enterprisewide capability will be a major undertaking, the idea here is to demonstrate a thread flowing between the conceptual and preliminary levels.

In the demonstration, the design process has only an interface to a conceptual design program. Thus, conceptual design is still somewhat autonomous from the overall design process. In order to effectively use high fidelity information with low level design parameters, the conceptual design process needs to be fully integrated with the overall design process. The same transition from interface to integration will take place with the preliminary and detailed design tools as well as the geometry module.

\section{THE ADAPTIVE MODELING LANGUAGE}

With dependency tracking, AML facilitates the control of a large number of design alternatives with a single set of driving requirements (feed forward). Dependency tracking can also be used to facilitate design parameterization (feed back). With demand-driven calculations, the designer can readily control when and how design information flows. AML already has built-in objects to address complex meshing and manufacturing issues. These capabilities, along with feature based geometry in a single open-access objectoriented architecture make AML very attractive as a means of addressing the complex design problem of a UAV.

Due to the weight of the conventional design process, we initially used AML as a means to establish interfaces between a number of design tools that have never before been interfaced. This presentation shows what can be accomplished with the interface approach.

Engineers in the Flight Dynamics Directorate have since discovered that AML will be much more powerful as a design integration tool if design objects are developed at a much finer level. For instance, we see merit in utilizing the geometric objects already available in AML, rather than interfacing with an existing CAD package. Another example is that we see the merit in selecting from a single suite 
of aerodynamic prediction objects, rather than the independent tools built into each of the design codes.

\section{THE DESIGN MODEL}

We have developed a generic Uninhabited Air Vehicle (UAV) for this demonstration. In order to aid in the exploration of a complex design process, it is in our interest to keep the design model as simple as possible.

The configuration used in this demo is shown in Figure (1). We are interested in the blended surface which is suitable for composite structures.

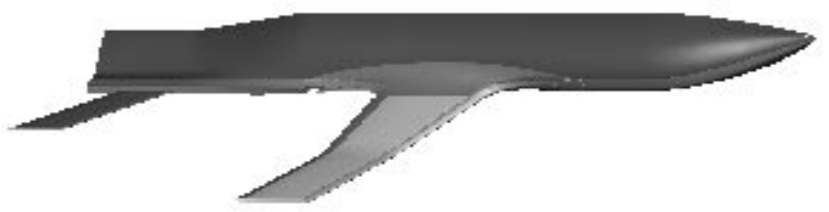

Figure (1) High Resolution Surface of UAV

The structure of the wing and center fuselage is addressed at the preliminary level [1]. The designer can specify the number of layers, orientation and the type of composite material used, then optimize the smeared stiffness properties (thicknesses) with aeroelastic loads.

The top wing skin is addressed at the detailed level. A solid FEM model will be developed, detailed stresses will be calculated based on loads developed at the preliminary level and the detailed structural part will be optimized.

\section{OVERVIEW of the DESIGN MODULES:}

Four design modules were selected for this demonstration of design feed forward and design feed back. A diagram of the implementation is given as Figure (2)

\section{CASP [2]:}

FUNCTION: Synthesize vehicle design requirements to carry out mission needs related to range and endurance. This conceptual module blends together database models of weights, aerodynamics and propulsion to generate design geometry. Subsequently, a predefined mission trajectory is simulated in order to calculate the fuel requirements. The designer modifies the vehicle design using CASP calculated design suggestions. The mission is simulated again and after a number of iterations, a balanced vehicle design is synthesized. CASP is not geometry based. Instead, simple geometric rules serve to compute volumes and areas.

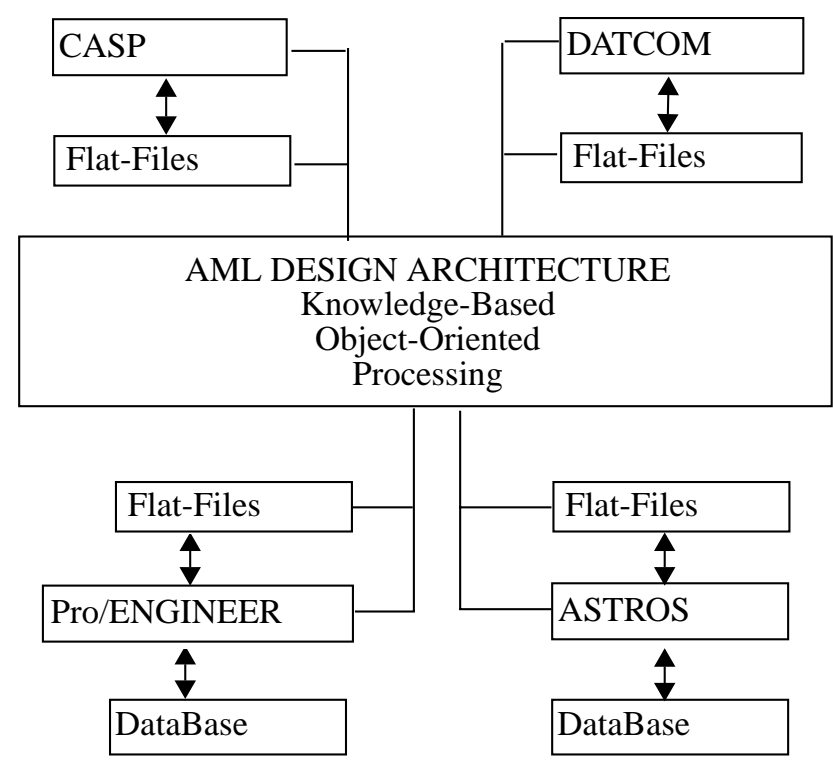

Figure (2) An Interfaced Design Process

AML DEVELOPMENTS: A Graphical User Interface (GUI) was developed for the functional areas of Geometry, Mission, Weights, etc. The Geometry GUI is shown in Figure (3). A conceptual geometric model of the current configuration keeps the designer informed. For the interface, flat files are constructed to move data into and out of AML. A design history file is retained. A restart capability allows the designer to save the design and pick up again later.

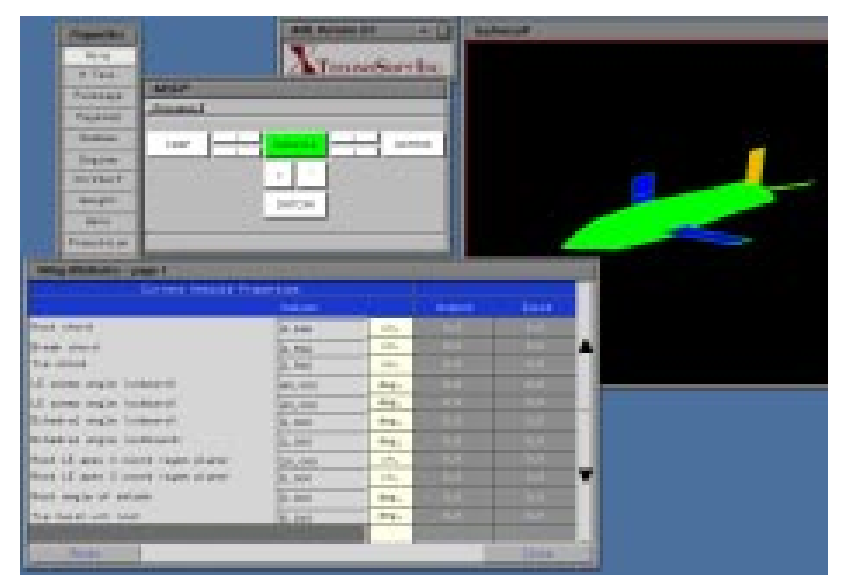

Figure (3) AML GUI Form for CASP Model

\section{DIGITAL DATCOM [3]:}

FUNCTION: Synthesize vehicle design requirements to carry out mission needs related to maneuverability. This conceptual design tool uses extensive data tables to synthesize vehicle designs with an emphasis on aerodynamics for stability and control. Preliminary level calculations create 
more accurate aerodynamic data to augment the data tables.

AML DEVELOPMENTS: A GUI was developed to inform the Digital Datcom user of the data coming from CASP and gives the designer the ability to select airfoil data. For the demonstration, AML is used to generate input and spawn a Digital Datcom process. Digital Datcom is used to generate an alternate table of drag polars for use in CASP. Regardless of whether Digital Datcom is the aerodynamic tool of choice to work with CASP, the idea is to demonstrate the ability to pass data in a feed forward and feed back design process.

\section{$\underline{\text { PRO/ENGINEER }}^{\text {TM}}$ :}

FUNCTION: This fully associative geometric model module will generate starting surface geometry for the ASTROS structural optimization module. We developed the capability to update dimensions in the Pro/ENGINEER model and regenerate a new geometric surface. Subsequently, the blended surface is extracted and the FEM surface mesh is parametrically updated. A new ASTROS FEM is generated. Figure (4) depicts a design version of the wing box and carry through structure.

AML DEVELOPMENTS: A GUI was developed to control the parametric structural thickness parameters. AML is used to take geometric variable data from the CASP design and pass it on to the Pro/E geometric model. Next, AML spawns the surface and mesh regeneration process.

\section{ASTROS [4]:}

FUNCTION: This module addresses the optimization of aerospace structures with a comprehensive model. The thickness of two dimensional (shell surface) elements are resized to generate the minimum (structural) weight design with multiple sets of aeroelastic loading conditions.

AML DEVELOPMENTS: A GUI was developed for setting up the executive and case control parameters. AML spawns the ASTROS process with data passed from GEO_FEM, a specialized mesh utility. After ASTROS arrives at the minimum weight design, AML reads the ASTROS database. High fidelity structural weights data are passed back to CASP for further design work. AML displays the FEM model with thickness and stress information.

\section{PRELIMINARY STRUCTURAL MODEL}

GEOMETRIC MODEL: The configuration for this design development is an uninhabited air vehicle (UAV) shown in Figure (1). While it does not make sense to list the dimensions of this fully associative model, it does help to describe the topology. This is a lambda wing concept, so the inboard portion of the wing can be made arbitrarily thick for addi- tional volume and the outboard portion is still thin and flexible. There is an internal surface for a weapons bay.

STRUCTURAL COMPONENTS: In order to focus our design process, we arbitrarily separated the wing box and center fuselage from the front and rear portions of the fuselage. The surface of this structural "box" is shown in Figure (4). Eventually, a more complete design will consider the entire vehicle. However, heaping on additional details does not help us develop the design process itself.

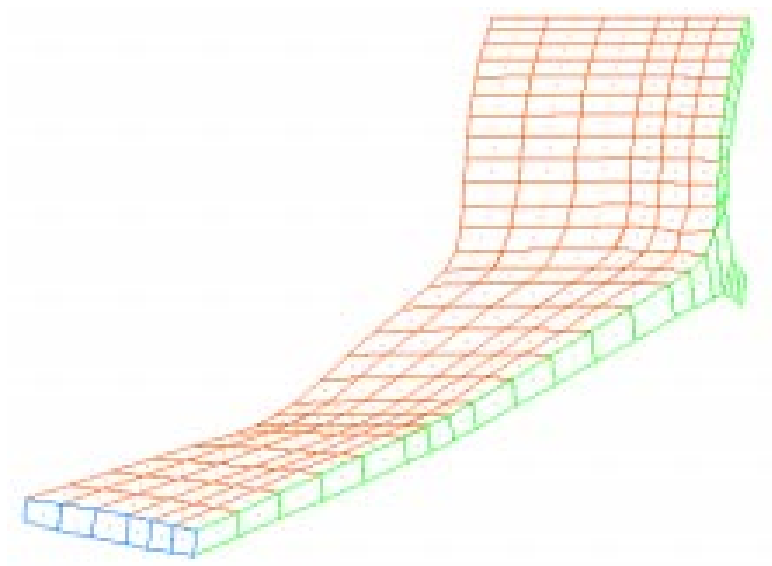

Figure (4) Finite Element Mesh

The wing leading and trailing edges have also been removed. All that remains is the primary wing box structure and fuselage carry-through. This model is useful for studying the primary load-paths. The model is cantilevered at the top along the plane of symmetry.

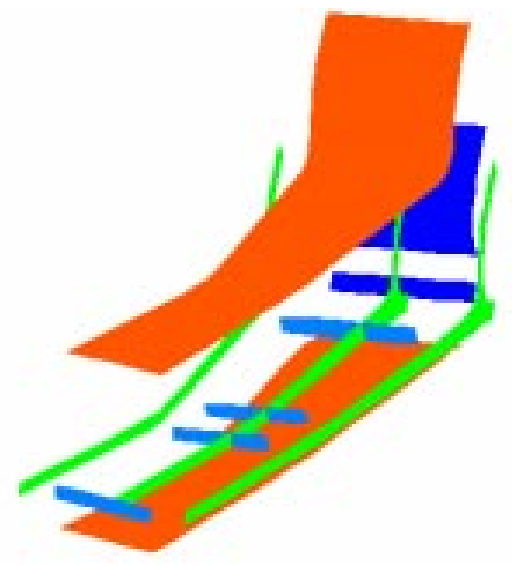

Figure (5) Exploded View

STRUCTURAL PARTS: There are a total of 11 parts in the structural model as shown in Figure (5). These parts include the top and bottom skins, three wing spars, four ribs, the fuselage inner bay, and the bay door jam. The entire component is designed with shell structures. The material selec- 
tion is whatever the conceptual designer would have us work with. For the example at hand, the top and bottom surfaces are anisotropic composite laminates. The remaining structure is quasi-isotropic. Each of the eleven structural parts were meshed with two-dimensional shell elements. The assembled mesh is depicted in Figure (4). This coarse mesh is suitable for INTERMEDIATE level design.

STRUCTURAL OPTIMIZATION: ASTROS is used to perform a MultiDisciplinary Optimization (MDO) to resize the thickness of the structural elements, and then to update the solid model representation. The conceptual designer may be interested in a design trade-off between structural weight and aerodynamic drag as the wing thickness is varied. For such a design study, the structural weights data generated by ASTROS is important.

ASTROS integrates a number of potentially conflicting design constraints such as material stress and aeroelastic flutter and converges on the optimal set of structural design variables to meet an objective function (e.g. minimum composite material weight). ASTROS is unique with its ability to achieve a single optimal structural design for a number of flight conditions involving various maneuvers at various speeds and altitudes. ASTROS uses a suite of one and two dimensional structural finite elements (e.g. beams, membranes and shells) which are tailored to the needs of aerospace designers who primarily work at the preliminary design level. ASTROS aerodynamic utilities address linear steady, unsteady, subsonic and supersonic conditions.

Linear aeroelastic loads are generated within ASTROS for the wing alone. ASTROS is used to resize the aeroelastic structure for minimum weight and simultaneously withstand the stress induced by a 6-g pull up (six times acceleration due to gravity). Tsai-Wu stress constraints and ply minimum gage constraints were applied [1].

\section{DESIGN PROCESS}

Designers have made a significant investment into independent CAD, CAM and CAE design software. These independent design software utilities have had a tremendously beneficial effect. However, making the data links between these high level modules is time consuming. We can spend almost as much time developing the links as developing the models themselves. Furthermore, the creation of a meaningful design process is complex and should be flexible. The overall design process takes place at a higher level than the design utilities (or modules).

The design process should be independent of the design modules (CAD, CAM and CAE). The designer should not apriori allow any one design tool to dictate the design process. For instance, if one allows FEM to drive the design, the designer may miss key geometric modeling issues. If one allows geometry to drive the design, the designer may overlook some subsystems functionality issues.

In Figure (2), the simple schematic showed how the various high level design modules were interfaced with the AML design architecture. Each module retains its own database and exchanges information with that database alone. Data flow from one design tool database to another is controlled in the AML design architecture. In this way, the design architecture has access to all necessary data. (However, with module interfacing, the design architecture cannot control what these high level modules do with the data.) Bidirectional data flow is facilitated by the design architecture. The modules in Figure (2) are somewhat autonomous and therefore results in a design process which is not wellprepared to address unanticipated synergistic effects of technologies. Ultimately, we want to work toward an integrated design process. However, before we can understand the integration issues, we have to understand the interface design process of Figure (2). This paper is a demonstration of interface-based design process.

\section{DEMONSTRATION:}

The demonstration assumes the baseline interface model already exists in Pro/ENGINEER and in AML for each of the analysis modules. With all the data connections in place, the resizing process was automated to arrive at a globally optimum solution in CASP using high fidelity structural weight generated by ASTROS.

Once all the data is in place in the architecture and all the interfaces are in place, the design process begins with the CASP program to size the vehicle fuel capacity (and all the geometric consequences) to meet mission range requirements. The CASP code alone develops a concept that requires $1500 \mathrm{lbs}$. of fuel. This data is returned to AML and the model is updated.

While the Digital Datcom code is normally used to resize all the aerodynamic surfaces to achieve maneuverability targets, we only used the aerodynamic portion related to wing lift. This aspect of the process will be revisited and enhanced when maneuverability is seen to drive the design.

Starting with a resized wing within AML, the high fidelity geometric model of the wing surface and structural parts are updated in Pro/ENGINEER by means of a flat-file with updated parameters. An auxiliary process with Pro/ DEVELOP, was automatically initiated to extract the surface geometry for all the wing parts. These parts were remeshed using the same parametric instructions as for the unsized model.

An ASTROS optimization process is then automatically initiated and the design is resized for minimum structural 
weight with the 6-g pull-up loads imposed. This weights data for an aeroelastically tailored design would never be found in a design handbook. The multidisciplinary variables used to drive the structural concept are too extensive. This high-fidelity weight data is passed back to AML.

Subsequently, the updated structural weight is used in a new CASP analysis. It turns out that the fuel weight only varies by ten pounds.

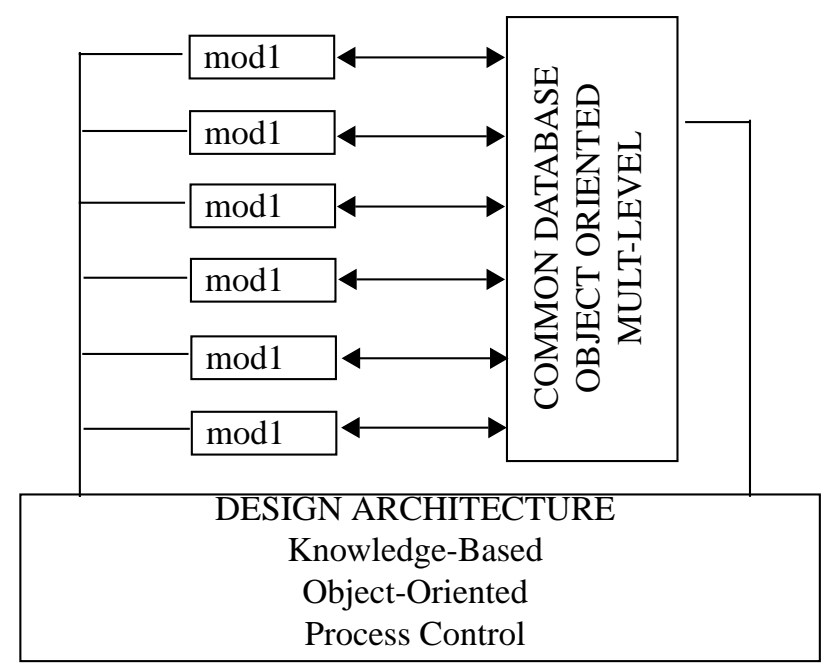

Figure (6) An Integrated Design Process

\section{TECHNOLOGY DIRECTION}

The purpose of this demonstration of design interface technology is to share our experience. For our team, the model was quite complex. Still, the design process presented here falls far short of a complete design process. However, a number of lessons were learned without incurring a huge expense.

The fact that the resized design model only shaved off ten pounds of fuel does not detract from the significance of the design model. As we incorporate elements of cost, producability, survivability (etc.), we will truly begin to see the payoff for feed forward and feed back. Ultimately, this leads to the capability to rapidly perform high fidelity cost - performance trades at the conceptual level.

We suggest that as the conceptual design tool (module) is disassembled and modularized there will be a motivation to migrate from the interface design process of Figure (2) to an integrated design process shown in Figure (6). This design system involves a number of disciplines with a number of software programmers. There needs to be some way for them to all share voluminous data. A common design database should be accessible by the knowledge based system and the analysis modules. Alternatively, we could work with a number of component databases driven by the same library of database calls. The component databases could actually be provided by different vendors, but there would be a requirement to establish some common standard calls (synonymous to the interactive SQL standards). In all cases, we should be able to see that in order to share data, there has to be some common mechanism.

The fuzzy vision of Figure (2) is something which designers talk around but has not been efficiently automated. The vision of Figure (2) is something which a number of designers are currently doing and with AML can be automated to the extent that the designer desires. Integration is where the design process has to go before we can really take advantage of concurrent design automation.

\section{COLLABORATIVE ENTERPRISES}

In developing a product, it is important that designers exercise rapid control of a high fidelity model. It is clear to the authors that AML facilitates such a design model with a wide range of metrics. With design intent firmly established in an AML model, the designer needs to gather high fidelity data from a number of sources. For an airplane design, these other sources of data might come from engine manufacturers or radome manufacturers or composite material manufacturers etc.

AML has a number of unique capabilities such as "demanddriven calculations", "dependency-backtracking" etc. These capabilities are not available in existing data-sharing standards. But these capabilities are important to the designer.

We forsee a collaborative enterprise, whose memebers agree to share designs based on AML technologies. AML objects are placed on the network by the producers. These objects describe their product (engine, radome, composite process etc) in a form which can be customized. The motivation toward such a collaboration will become strong when participants discover a large decrease in the design time of high fidelity models.

\section{REFERENCES}

[1]Max Blair, Geetha Bharatram, Robert A. Canfield, "Designing a Blended Composite Wing and Fuselage" AIAA Paper 96-3995, presented at the 6th AIAA/NASA/ISSMO Symposium on MultiDisciplinary Analysis and Optimization, 4 September 1996.

[2]B. H. Oman, S. K. Pederson, N. P. Karll, T. F. Reed, "Computer Program to Perform Aircraft Design Synthesis, AFFDL-TR-7435 .

[3]J. E. Williams, and S. R. Vukelich, "The USAF Stability and Control Digital Datcom,” AFFDL-TR-79-3032, April 1979.

[4]D. J. Neill and D. L. Herendeen, "ASTROS Enhancements", WL-TR-96-3004 (Vol I: “ATROS User's Manual”), WL-TR-963005 (Vol II: “ASTROS Programmer's Manual”), WL-TR-953006 (Vol III: “ASTROS Theoretical Manual”). 